\title{
Obesity's impact on survival is independent of dose adjustments in neoadjuvant chemotherapy in women with breast cancer
}

\author{
Ying L. Liu ${ }^{1}$ Eileen P. Connolly ${ }^{2} \cdot K^{2}$ in Kalinsky ${ }^{2}$
}

Received: 17 November 2017 / Accepted: 22 November 2017 / Published online: 24 November 2017

○) Springer Science+Business Media, LLC, part of Springer Nature 2017

To the Editor,

Dr. Altundag's comment brings to attention an important question about whether part of obesity's negative impact on survival in women with breast cancer receiving adriamycin/taxane-based chemotherapy is due to dose reduction in the cytotoxic systemic therapy. They cite a recent study by Karatas et al. in which chemotherapy doses were capped at a body surface area (BSA) of $2 \mathrm{~m}^{2}$ and were not uniformly based on a patient's exact BSA, potentially contributing to obesity's negative impact on survival [1]. In our study [2], we also evaluated this concept. Of the 273 women included in our study, 10 patients had high BSA $(\geq 2.2)$ and 14 were morbidly obese [body mass index (BMI) $\geq 40$ ], but none of these women had reductions in their chemotherapy doses. In addition, there were no differences in the type of surgery (lumpectomy vs. mastectomy) or radiation therapy rates as compared to women with normal weight (BMI 18.5 to $<25$ ) and BSA $<2.2, p>0.05$. This suggests that obesity's negative impact on survival is independent of chemotherapeutic dosing.

\section{References}

1. Karatas F, Erdem GU, Sahin S et al (2017) Obesity is an independent prognostic factor of decreased pathological complete response to neoadjuvant chemotherapy in breast cancer patients. Breast (Edinb Scotl) 32:237-244

2. Liu YL, Saraf A, Catanese B et al (2017) Obesity and survival in the neoadjuvant breast cancer setting: role of tumor subtype in an ethnically diverse population. Breast Cancer Res Treat. https:// doi.org/10.1007/s10549-017-4507-y
This is a letter to the editor submitted in response to Dr. Kadri Altundag's letter to the editor titled "Dose adjustment in obese breast cancer patients receiving neoadjuvant chemotherapy might have an impact on survival" which was published by this journal online on October 23rd, 2017.

Kevin Kalinsky

kk2693@columbia.edu

1 Memorial Sloan Kettering Cancer Center, New York, NY, USA

2 New York Presbyterian Hospital, Columbia University Medical Center, New York, NY, USA 